\title{
Alcance Geoespacial de Atención del Cuerpo de Bomberos. El Caso del Municipio de Manizales en Colombia
}

\author{
Diego A. Escobar(1)*, Santiago Cardona ${ }^{(1)}$ y Carlos A. Moncada(2) \\ (1) Facultad de Ingeniería y Arquitectura, Departamento de Ingeniería Civil, Universidad Nacional de Colombia, \\ Sede Manizales, Carrera 27 \# 64 - 60, Campus Palogrande, Bloque Posgrados, Oficina I - 408, Colombia. \\ (e-mail: daescobarga@unal.edu.co; scardonau@unal.edu.co) \\ (2) Facultad de Ingeniería, Departamento de Ingeniería Civil y Agrícola. Universidad Nacional de Colombia, \\ Sede Bogotá, Ciudad Universitaria, Edificio 214, Oficina 325, Colombia. (e-mail: camoncadaa@unal.edu.co) \\ * Autor a quien debe ser dirigida la correspondencia
}

Recibido Mar. 8, 2019; Aceptado Abr. 25, 2019; Versión final May. 17, 2019, Publicado Oct. 2019

\begin{abstract}
Resumen
Las estaciones de bomberos, como eje primordial en el proceso de protección de personas y bienes, deben garantizar el menor tiempo de reacción frente a una eventual emergencia. Por lo tanto, se deben realizar procesos de análisis de cobertura, localización y acceso, que permitan seleccionar el sector más apropiado para su implementación. En esta investigación, se propone un análisis, mediante accesibilidad territorial, que permita evaluar la cobertura de las estaciones de bomberos en la ciudad de Manizales, en Colombia, a través de la red de infraestructura de transporte, bajo características de operativas actuales, con el fin de localizar áreas con falencias en los tiempos mínimos de respuesta recomendados y su relación con la equidad social y cobertura. Existe inequidad en la cobertura del servicio, pues se encontró que los estratos sociales 1 y 2 (baja capacidad económica), son los que menores porcentajes de cobertura refieren para un tiempo mínimo de respuesta de seis minutos con $7 \%$ y $1 \%$, respectivamente, mientras que para los estratos 5 y 6 (alta capacidad económica), los porcentajes de cobertura para igual período de tiempo son de $28 \%$ y $35 \%$, respectivamente.
\end{abstract}

\section{Geospatial Extent of a Fire Department Attention. The Case of the Municipality of Manizales in Colombia}

\begin{abstract}
The fire stations, as the main axis in the process of protection of people and goods, must guarantee shortest reaction time in the event of an emergency. Therefore, coverage, location and access analysis processes must be carried out to select the most appropriate sector for its implementation. In this research, an analysis is proposed, through territorial accessibility, which allows to evaluate the coverage of fire stations in the city of Manizales in Colombia, through transport infrastructure network, under current operating characteristics, in order to locate areas that have shortcomings in the recommended response times and their relationship with social equity and coverage. Results show that there is an inequality in service coverage; a social strata 1 and 2 (low economic income) have lower percentages of coverage for a minimum response time of six minutes with $7 \%$ and $1 \%$, respectively, while that for strata 5 and 6 (high economic income), the percentages of coverage for the same period of time are $28 \%$ and $35 \%$, respectively.
\end{abstract}

Keywords: territorial accessibility; fire stations; emergency; coverage; road network 


\section{INTRODUCCIÓN}

Actualmente, dadas las infinitas posibilidades de acopiar información geográfica relacionada con la ubicación de equipamientos y actividades, no solo permiten la realización de análisis de estructura urbana, usos del suelo y características operativas de las infraestructuras de transporte. Sino también se convierte en una oportunidad para la adecuada planificación y gestión en la prestación de servicios mediante la aplicación de técnicas y metodologías que expresen cambios en los espacios geográficos (Moreno y Jácome, 2017). Es así que conocer y analizar la distribución geográfica de un servicio a la comunidad, permite definir políticas prioritarias de atención por parte de un gobierno. Por ejemplo, estudios enfocados en mitigar problemas de salud pública como el Síndrome de Abstinencia Neonatal (NAS acrónimo en inglés), han encontrado un variabilidad temporal y geográfica significativa y proporcional con las variaciones en la prescripción de Analgésicos Opioides (OPR acrónimo en inglés) en los Estados Unidos (Patrick et al., 2015), información que permitió a los gobiernos locales generar propuestas de intervención al respecto; proyectos como el desarrollado en Jakarta (Indonesia) el cual buscó mejorar la resiliencia de la ciudad a las inundaciones (Moreno y Jácome, 2017) aplicando análisis geográfico para la atención adecuada de las emergencias; estudios cuyo objetivo fue el identificar las necesidades de la población para alcanzar servicios como educación, salud y seguridad (Escobar et al., 2016).

Manizales (ver Figura 1), capital del Departamento de Caldas, se encuentra ubicada en el centro occidente de Colombia, cuenta con cerca de 420.000 habitantes en su área urbana, la cual se toma junto al municipio vecino de Villamaría debido a que forman un área metropolitana dadas las continuas dinámicas sociales entre sus habitantes. Manizales $(2.150 \mathrm{msnm})$ cuenta con una topografía abrupta. A pesar de esto, en términos de movilidad urbana, el modo de transporte que posee mayor preferencia es la caminata (29\%), seguido por el transporte público, aunque este último ha visto una disminución importante en los últimos años en el número de los pasajeros movilizados (Cardona et al., 2017), tendencia que sumada al crecimiento del parque automotor de vehículos privados, especialmente la motocicleta, genera un panorama difícil en términos de movilidad urbana en los próximos años para la ciudad.
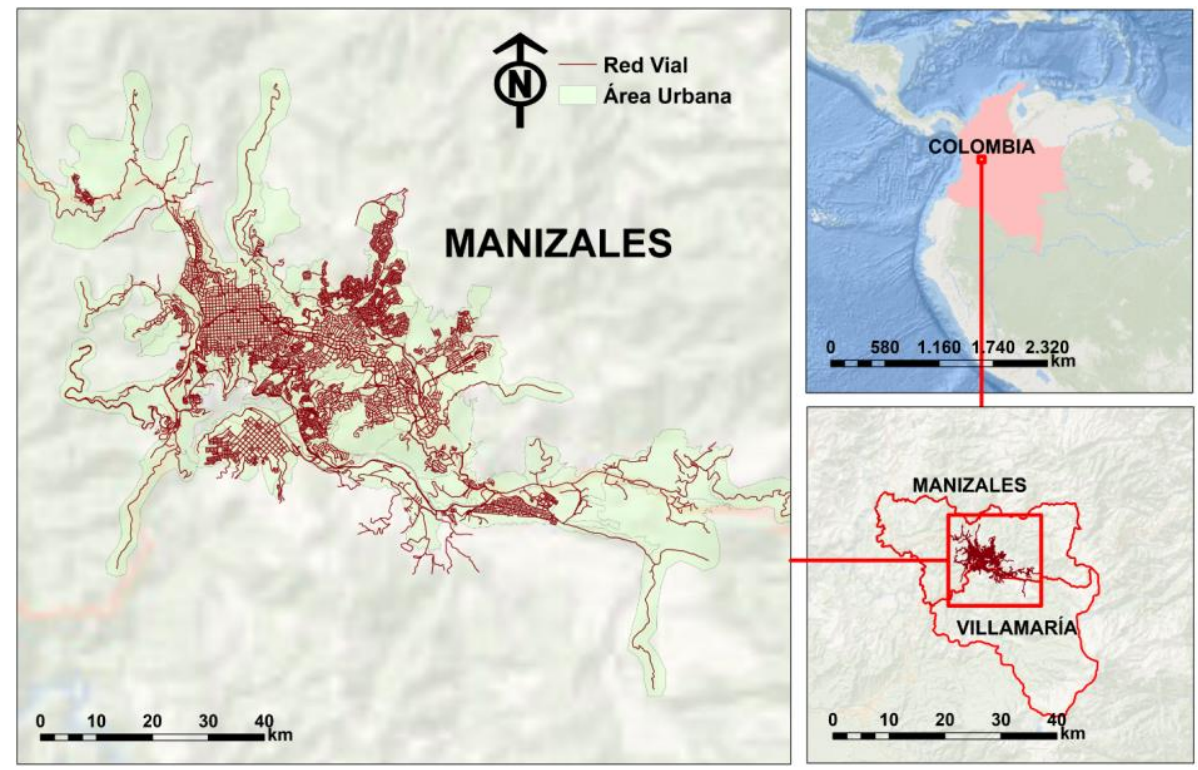

Fig. 1: Localización geográfica de la zona de estudio (Adaptado de ArcGis Online)

Por su parte, la principal misión de las estaciones de bomberos, como cuerpo de reacción inmediata, es el de brindar las herramientas necesarias para salvaguardar vidas y bienes de los diferentes fenómenos naturales y eventualidades que se puedan presentar (Lazo, 2017). Por ello, se hace de vital importancia, determinar el tiempo de respuesta generado por cada estación, a través de la red de infraestructuras del transporte. Hoy en día, existen diversas metodologías para cuantificar el área de cobertura que posee cada estación de bomberos en una ciudad; para esta investigación se analiza la accesibilidad territorial, la cual ha sido utilizada para calcular la población cubierta por sistemas de emergencia como los centros de ambulancia (Holguín et al., 2017). La accesibilidad territorial permite cuantificar la cobertura de diversos equipamientos a través de la red de infraestructuras del transporte, evaluando la oferta presente en una ciudad (Escobar et al., 2018). El término "accesibilidad", desde su primera definición, se establece como el potencial de oportunidades de interacción (Hansen, 1959), tomando diferentes enfoques (Geurs y Van Wee, 2004), perspectivas (Geurs y Ritsema, 2001), análisis (Ingram, 1971) y tipos de medidas (Pirie, 1979), caracterizándola como una metodología de suma importancia en la planificación urbana y del transporte que posee múltiples retos y 
aplicaciones (Geurs et al., 2015; Perilla et al., 2018; Van Wee, 2016). Diversos estudios se han realizado en la ciudad relacionados con la accesibilidad territorial, utilizándola como herramienta de planeación urbana para la evaluación de sistemas de transporte público (Escobar et al., 2015), universidades (Younes et al, 2016), centros comerciales (Zuluaga y Escobar, 2017), eco parques (Escobar et al., 2018), cobertura de equipamientos como los centros de ambulancia (Holguín et al., 2018), entre otros. En esta investigación se realiza un análisis de cobertura para las estaciones de bomberos en Manizales y su relación con la población, su nivel socio económico y el tiempo de respuesta esperado, obteniendo un análisis relacionado con la equidad social y la cobertura de equipamientos de emergencia (Holguín et al., 2018). Estos estudios, en la mayoría de las ocasiones, han tenido un componente evaluativo de la equidad social en el acceso al transporte debido a su importancia para cerrar brechas de desigualdad alrededor del mundo (Lucas et al., 2016).

\section{METODOLOGÍA}

En la Figura 2 se observa el diagrama de flujo de la metodología llevada a cabo en la investigación. En esta, se presenta una etapa previa donde se obtienen los datos necesarios para el cálculo, entre los que se cuentan la red de infraestructuras del transporte y el polígono de barrios de la zona de estudio. Estos datos fueron actualizados para investigaciones realizadas con anterioridad por lo que cumplen con los requisitos mínimos entre los que se cuenta la topología de la red y su cumplimiento con la teoría de grafos y la proyección de la población de Manizales hasta 2017 en el polígono de barrios (Cardona et al., 2018; Deo, 1974). Cabe aclarar, que las velocidades dispuestas en los arcos de la red de infraestructuras del transporte corresponden a datos reales obtenidos mediante sistemas de posicionamiento global (Younes et al., 2016).

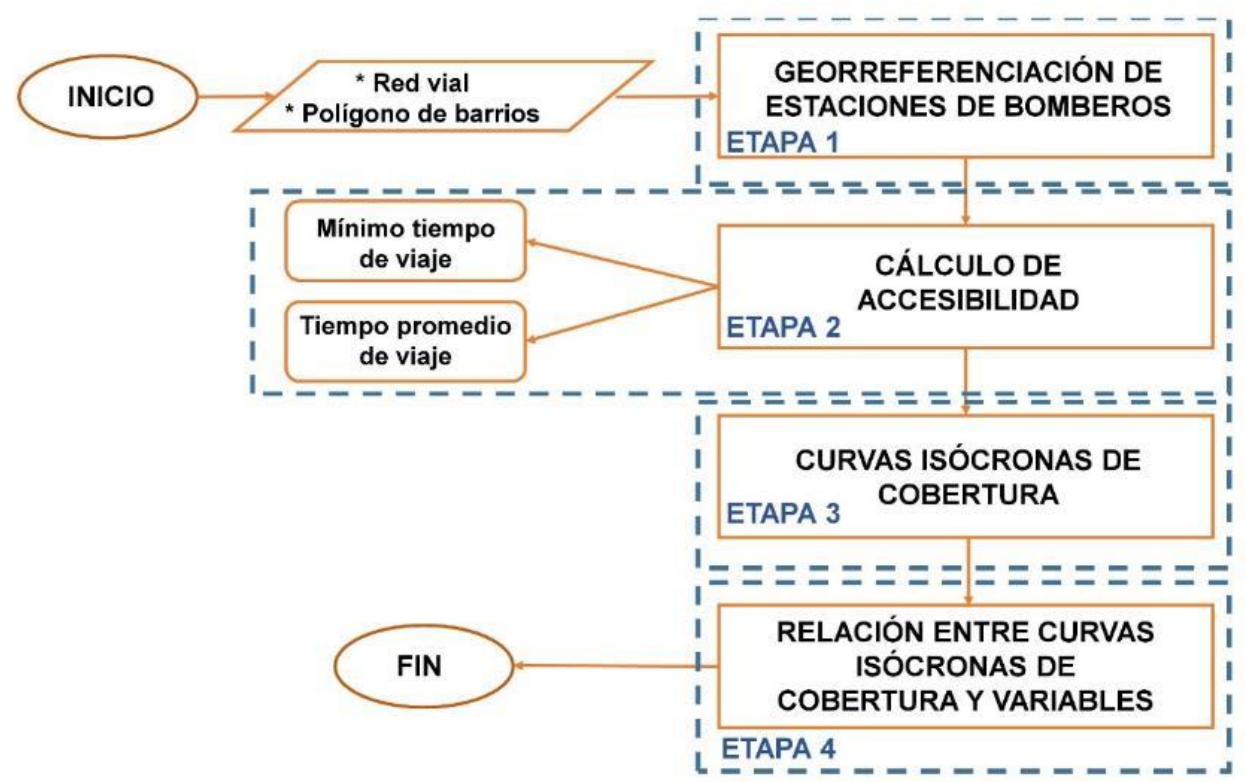

Fig. 2: Metodología de investigación desarrollada.

Etapa 1. Georreferenciación de las estaciones de bomberos: En la primera etapa se georreferencian las estaciones de bomberos existentes en la ciudad de Manizales y Villamaría, mediante el uso de sistemas de información geográfica (GIS), en este caso el software ArcGis (Escobar et al., 2018; Holguín et al., 2018). Asimismo, se debe relacionar el nodo de la red de infraestructuras del transporte más cercano a cada estación, con lo cual se podrán realizar los cálculos de accesibilidad territorial en una etapa posterior.

Etapa 2. Cálculo de accesibilidad: En esta etapa se realiza el cálculo de la accesibilidad territorial mediante la obtención del vector de tiempo medio de viaje $\left(T_{v i}\right)$, que expresa el tiempo medio de viaje desde un nodo i hasta los demás nodos de la red. Mediante la aplicación del algoritmo de Dkjistra del SIG, que permite calcular la menor impedancia (camino mínimo) entre un nodo específico (estación de bomberos) y el resto de nodos de la red, se obtiene la matriz de impedancias en términos de distancia; posteriormente, con los valores de velocidad de operación de la red de infraestructuras de transporte, se obtiene la matriz de tiempos medios de viaje, la cual en este caso de investigación, tiene un tamaño de $10.088 \times 4$ (Escobar et al., 2018), ya que actualmente existen 4 estaciones de bombero en servicio. Se analizaron dos niveles de cobertura, en primer lugar, se calculó el tiempo medio de viaje desde cada estación de bomberos $\mathrm{k}$, hasta cada nodo i de la red de infraestructuras del transporte, obteniéndose el vector de tiempo medio de viaje mediante la sumatoria de los tiempos de viaje desde cada una de las 4 estaciones y dividiendo entre el número de equipamientos (Ver Expresión (1); en segundo lugar, se analizó el tiempo mínimo de viaje desde las estaciones de bomberos a cada nodo de la red vial, lo que nos indica la estación de bomberos que puede responder en menor tiempo 
ante una emergencia, obteniendo se un vector de tiempos mínimos de respuesta (Ver Expresión (2)). Donde, $T_{v i}=$ tiempo de viaje mínimo promedio entre el nodo i y los demás nodos de la red; $\mathrm{n}$ = número de nodos de la red; $\mathrm{m}=$ número de estaciones de bomberos.

$$
\begin{gathered}
\overline{T_{v l}}=\frac{\sum_{k=1}^{m} t_{v_{k i}}}{m} \text { siendo } i=1,2,3, \ldots, n ; k=1, \ldots, \mathrm{m} \\
\text { min } t_{v_{k i}} \text { siendo } i=1,2,3, \ldots, n ; k=1, \ldots, \mathrm{m}
\end{gathered}
$$

Etapa 3. Curvas isócronas de cobertura: Una vez obtenidos los vectores de tiempos medios de viaje y tiempos mínimos de viaje, es posible construir las curvas isócronas de cobertura mediante un análisis geoestadístico, rama de la geografía que analiza un conjunto de datos en superficie (Matheron, 1963); estos métodos se encargan de predecir valores en el espacio mediante la entrada de datos, vectores de tiempo en el caso de la accesibilidad territorial. El método utilizado en diversas investigaciones dados sus buenos resultados es el Kriging Ordinario con semivariograma lineal (Oliver y Webster, 1990; Simpson et al., 2001). Este modelo nos permite obtener las curvas isócronas de coberturas para los dos escenarios analizados.

Etapa 4. Relación entre curvas isócronas de cobertura y variables: Por última, se realiza una comparación entre el polígono de barrios y las curvas isócronas de cobertura de ambos escenarios. Esto permite calcular la relación entre población, área urbana, estratos socioeconómicos y cobertura de las estaciones de bomberos (Perilla et al., 2018; Younes et al., 2016).

\section{RESULTADOS Y DISCUSIÓN}

Los resultados se presentan bajo tres aspectos: (i) georreferenciación de estaciones de bomberos, red vial y polígono de barrios; (ii) tiempo mínimo de respuesta y cobertura para las estaciones de bomberos; y (iii) tiempo promedio de respuesta y cobertura para las estaciones de bomberos.

Georreferenciación de estaciones de bomberos. En la Figura 3 se puede observar la localización de las cuatro (4) estaciones de bomberos operativas en Manizales y Villamaría, está información fue obtenida a partir de diversas fuentes de datos y verificada mediante llamadas telefónicas a cada una de las estaciones con el fin de confirmar su ubicación. Así mismo, el polígono de barrios, el cual cuenta con 150 barrios, incluyendo Villamaría y corresponde al área de estudio, la cual alcanza 5.868 hectáreas y una población de 419.944 personas. Además, se observa la red vial la cual está compuesta por 12.714 arcos y 10.088 nodos.

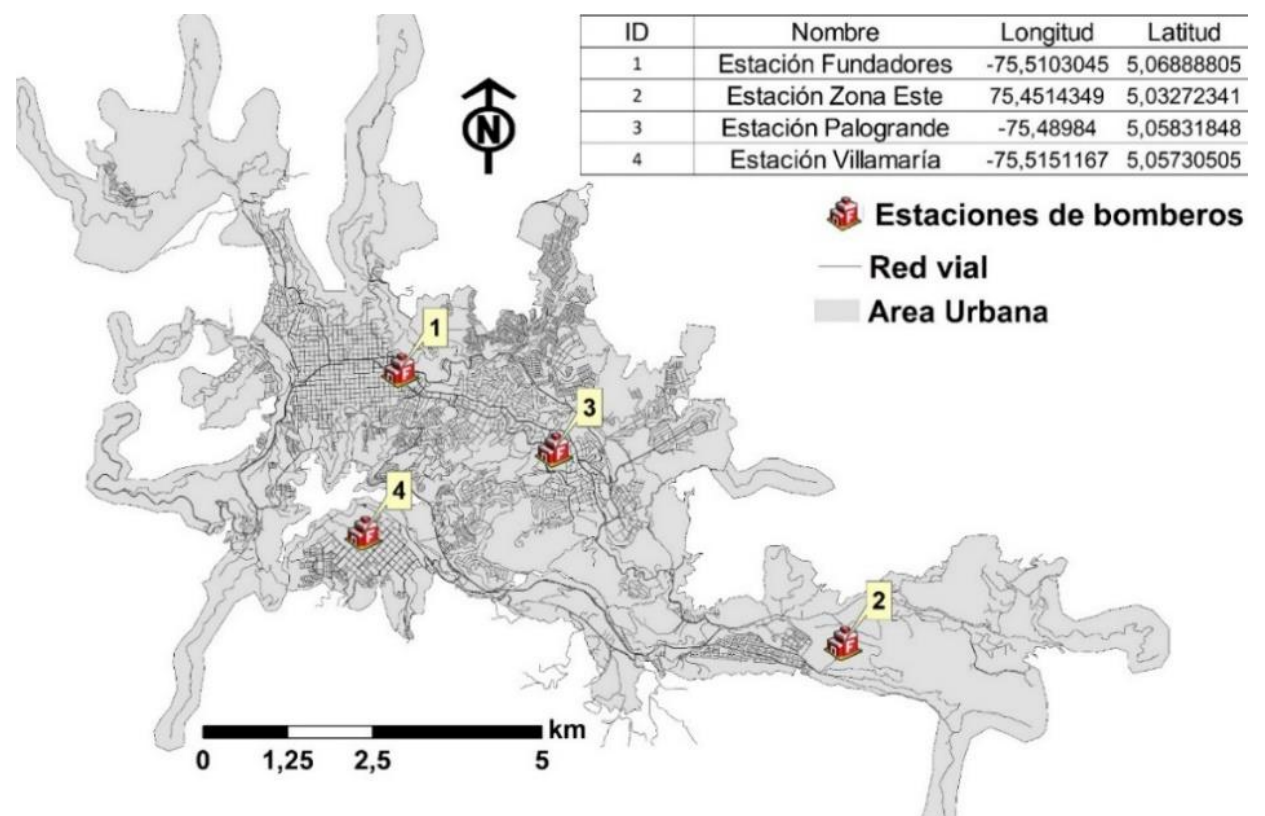

Fig. 3: Estaciones de bomberos, red vial y polígono de barrios.

Tiempo mínimo de respuesta y cobertura. En la Figura 4 se puede observar el resultado del análisis de cobertura de las estaciones de bomberos en su tiempo mínimo de respuesta, es decir, teniendo en cuenta el nodo más cercano de la red vial a cada estación de bomberos en la zona de estudio. En estás, se puede apreciar como existe un tiempo mínimo de respuesta de 0.59 min y un tiempo máximo de 45 minutos. Este último dato corresponde a áreas del polígono estudiado donde no se tienen registros de población por lo que realmente el $100 \%$ de la población está cubierto por tiempos menores a 30 minutos, lo cual puede observarse 
en la Figura 5a que corresponde a las ojivas de porcentaje acumulado de población y área para este escenario. Así mismo, se aprecia que el $10 \%$ de la población se encuentra dentro del tiempo mínimo de respuesta de 5 minutos o menos, porcentaje que crece hasta un 48\% para un tiempo mínimo de respuesta de 10 minutos o menos. Por otra parte, para cada curva isócrona de cobertura, el porcentaje de población es más alto que el del área urbana debido a que gran cantidad de población se concentra en las áreas centrales de la ciudad. En la Figura $5 \mathrm{~b}$ se observa el análisis de cobertura poblacional según los estratos socioeconómicos. Al ser un análisis de población, los tiempos más altos de cobertura alcanzan los 30 minutos. Se observa que los estratos de mayor nivel socio económico, estrato 5 y 6 , poseen una mejor cobertura de las estaciones de bomberos en la ciudad de Manizales, logrando un 50\% de cobertura para un tiempo de entre 5 y 10 minutos; de otro lado, los estratos bajos, estrato 1 y 2, poseen una cobertura deficiente por lo que los tiempos de respuesta a estos lugares y a esta población es más alta, rondando los 14 minutos para lograr el mismo valor de cobertura.

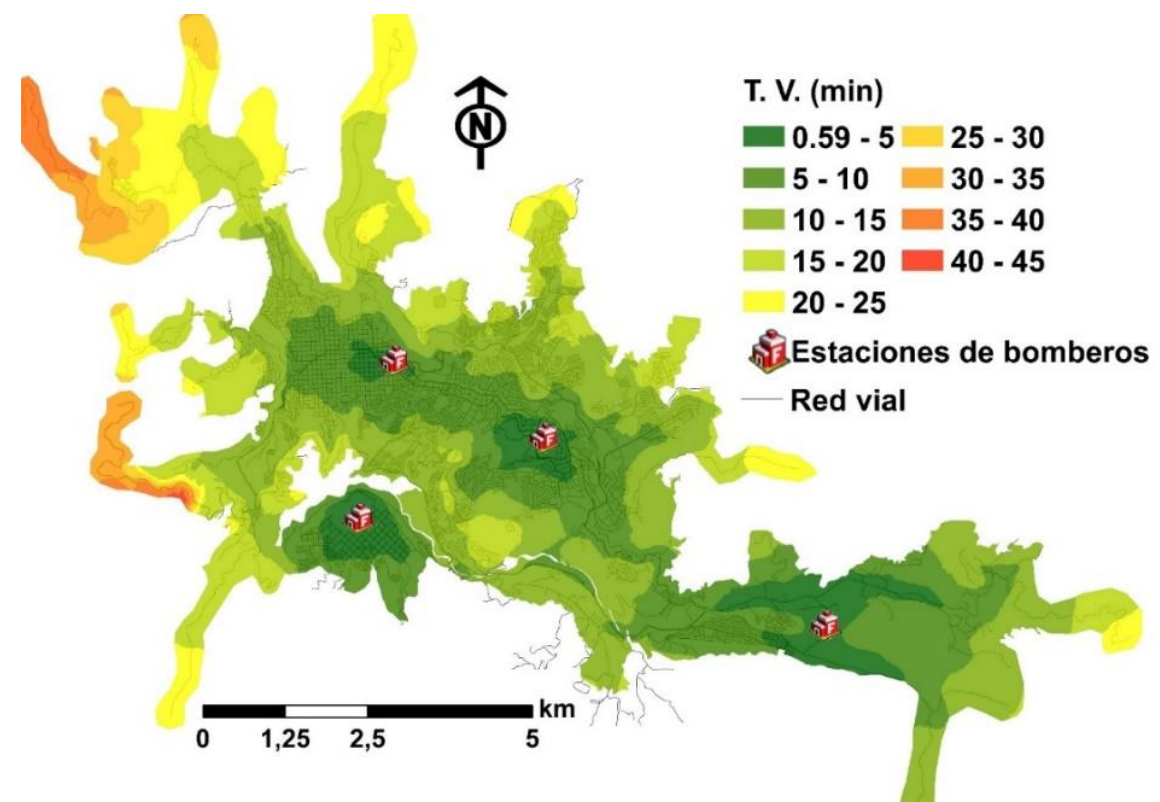

Fig. 4: Curvas isócronas de cobertura para el mínimo tiempo de viaje desde las estaciones de bomberos
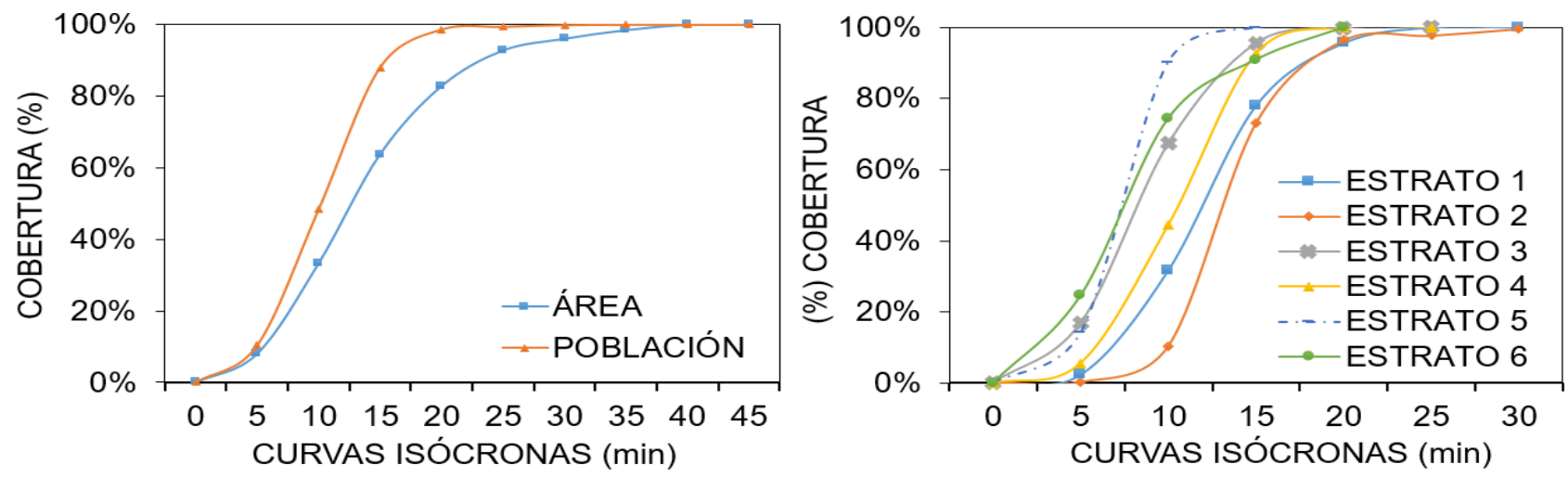

Fig. 5: Relación entre curvas de tiempo mínimo de respuesta y las variables a) Población, Área; b) Población según estrato socioeconómico.

Tiempo promedio de respuesta y cobertura. En este análisis se optó por realizar la sumatoria de los tiempos de viaje de todas las estaciones a cada nodo para posteriormente dividirlo entre el número de estaciones de bomberos analizadas. Esto nos servirá de indicativo de la cobertura ante una presunta emergencia que requiera la acción de todas las estaciones de bomberos de la ciudad. En la Figura 6 se observa cómo el tiempo promedio de respuesta para las 4 estaciones de bomberos es de 15 minutos y el tiempo máximo es de 53,67 minutos, el cual se presenta en áreas de la ciudad donde no se tienen registros de población. Por otra parte, el tiempo máximo de respuesta para barrios donde se tienen registros de población es de 40 minutos; así mismo, en la Figura 7a se observa que el $99 \%$ de la población está cubierta por tiempos promedio de viaje de 30 minutos o menos. Para tiempos promedio entre 15 y 20 minutos existe una amplia cobertura de población que alcanza un $49 \%$. En el análisis que corresponde a estratos socio económico de población (ver Figura $7 b$ ) se sigue la tendencia generada en el escenario anterior donde los estratos con mejor nivel poseen una cobertura de tiempos mejor respecto a los estratos socio económicos de menos recursos. 


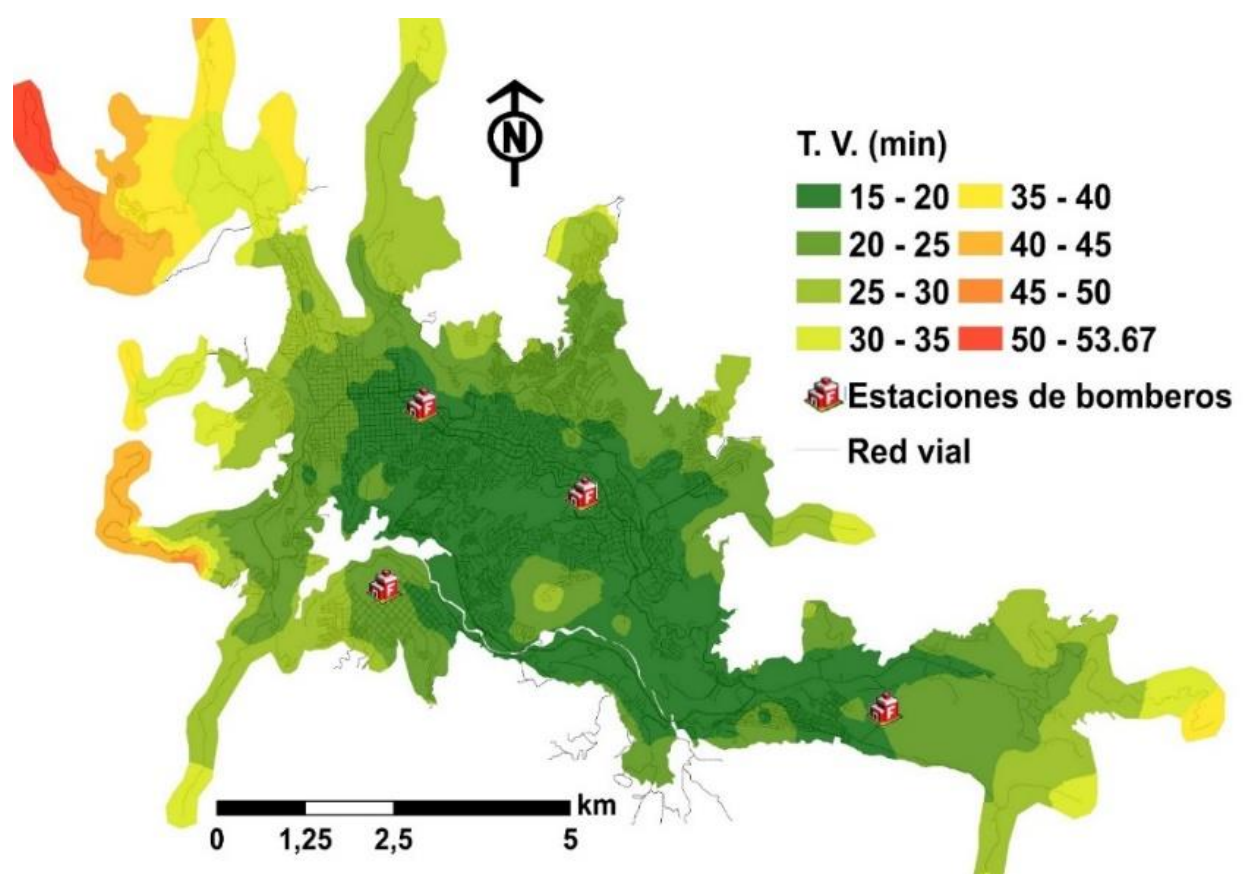

Fig. 6: Curvas isócronas de cobertura para tiempo promedio de respuesta desde las estaciones
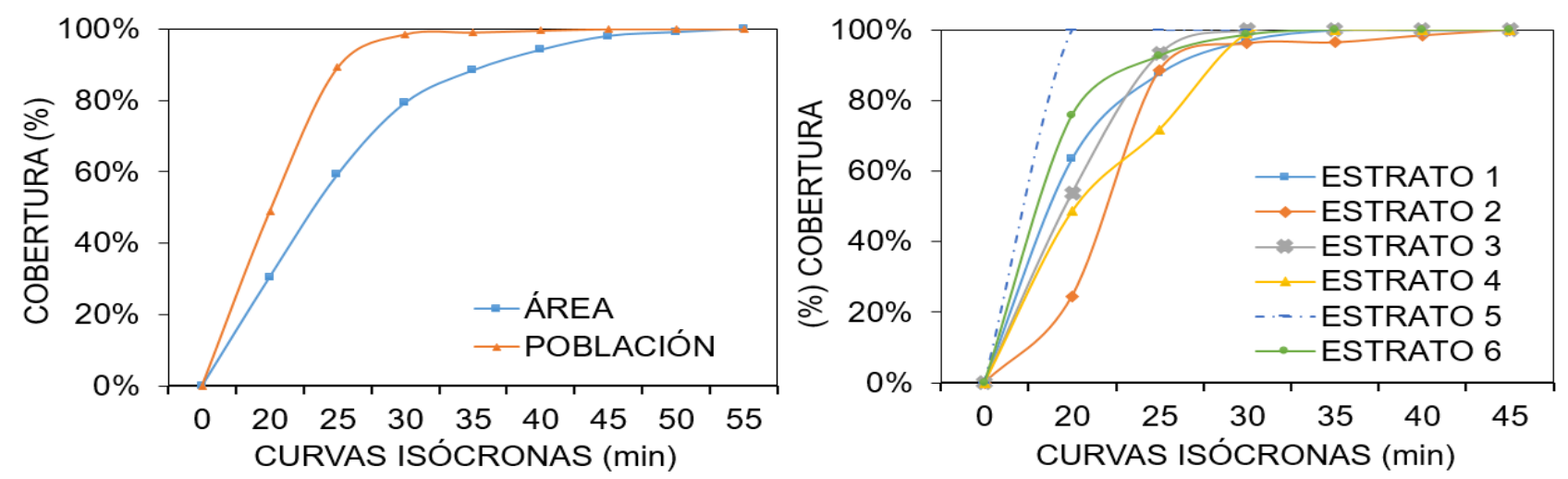

Fig. 7: Relación entre curvas de tiempo promedio de respuesta y las variables a) Población, Área; b) Población según estrato socioeconómico.

\section{DISCUSION FINAL}

Variadas investigaciones realizadas sobre el tema han establecido tiempos de respuesta de una estación de bomberos, por ejemplo, según la "National Fire Protection Association" el tiempo de respuesta debe ser máximo de 6 minutos ( 1 minuto para la confirmación del evento, 1 minuto para la preparación y 4 minutos para el desplazamiento) (Gallego y Suárez, 2015); la Defensa Civil de Singapore (SCDF acrónimo en inglés) propone como tiempo de respuesta máximo 8 minutos, definiendo como ideal 6 minutos (Liu et al., 2006); en Kuwait han establecido que el tiempo de respuesta óptimo es de 5 minutos (Savsar, 2014); en Nasiriyah (Iraq) han propuesto un tiempo de respuesta máximo de 4 minutos (Murtadha et al., 2018); investigaciones de análisis de localización óptima con algoritmos genéticos han usado un tiempo de respuesta de 5 minutos (Zhang y Jiang, 2012). Se observa entonces que los tiempos máximos de respuesta varían entre 4 y 6 minutos.

En la investigación realizada por Zhang y Jiang (2012) se analizó la cobertura de seis estaciones de bomberos en un área de $36 \mathrm{Km}^{2}$, situación bastante similar a la de este estudio, encontrando que con el planteamiento propuesto por ellos se lograba una cobertura del $67 \%$ de la población para un tiempo de respuesta de cinco minutos. Por su parte, la investigación llevada a cabo en Nasiriyah (Iraq), con cinco estaciones de bomberos se alcanzó una cobertura del $54 \%$ de la población en un tiempo de respuesta de 4 minutos para una población de 800 mil habitantes (Murtadha et al., 2018). Así mismo, en la investigación realizada en Singapore (Liu et al., 2006) con 22 estaciones de bomberos se alcanzó una cobertura poblacional superior al $60 \%$ en un tiempo de respuesta de seis minutos para una población cercana a los 5 millones de habitantes. Finalmente, la investigación realizada en Kuwait (Savsar, 2014) analiza la ubicación óptima de las estaciones de bomberos según los incidentes registrados y la población, se propone una metodología de ubicación de nuevas estaciones de bomberos en un tiempo de respuesta de máximo 5 minutos, pero no analiza la cobertura poblacional de las mismas. Por su parte, Manizales, con un área aproximada de $36 \mathrm{Km}^{2}, 400$ mil habitantes y 
custro estaciones de bomberos, para tiempos de respuesta de entre 4 y 6 minutos se tienen coberturas poblacionales de tan sólo el $8 \%$ y $18 \%$, respectivamente, valores bastante distantes a los porcentajes observados en las otras ciudades mencionadas. Para lograr una cobertura del $60 \%$ de la población en Manizales, se deben invertir desde las estaciones de bomberos actuales unos 11 minutos de tiempo mínimo de viaje en promedio, lo cual es casi el doble de lo definido como óptimo por la "National Fire Protection Association" y la Defensa Civil de Singapur.

Así que, para cumplir con las normas internacionales deberían adecuarse nuevas estaciones de bomberos a lo largo de la ciudad con el fin de aumentar la cobertura de población en el tiempo mínimo de respuesta. Vale la pena resaltar las condiciones de inequidad observadas en esta investigación en relación con la cobertura de los diferentes estratos sociales dada la ubicación de las estaciones de bomberos y las características operativas de la red de infraestructuras del transporte, pues se encontró que son precisamente los estratos sociales 1 y 2 (baja capacidad económica), los que menores porcentajes de cobertura refieren para un tiempo mínimo de respuesta de seis minutos con $7 \%$ y $1 \%$, respectivamente, mientras que para los estratos 5 y 6 (alta capacidad económica), los porcentajes de cobertura para igual período de tiempo son de $28 \%$ y $35 \%$, respectivamente.

\section{CONCLUSIONES}

De acuerdo al trabajo presentado y a los resultados obtenidos, se pueden plantear las siguientes conclusiones principales:

1.- Dados los resultados de cobertura obtenidos mediante el análisis geoestadístico, se concluye que existen tres sectores de la ciudad con la necesidad de que se ubique una nueva estación de bomberos en sus inmediaciones, son el sector noroccidente, suroccidente y norte. La definición más precisa de ubicación de estas tres estaciones de bomberos, así como el estudio del impacto sobre la cobertura poblacional se configuraría como una línea de investigación complementaria.

2.- Dadas las condiciones de inequidad encontradas en la cobertura poblacional, dada la ubicación de las estaciones de bomberos y las características operativas de la red de infraestructuras del transporte, se recomienda la ubicación de nuevas estaciones de bomberos en barrios cuyos estratos socio económicos sean bajos (estratos 1 y 2). Por otro lado, en el tiempo promedio de cobertura, para los eventos que requieren atención de todas las estaciones de bomberos también existe una disparidad social, aunque el $50 \%$ de la población está cubierta por tiempos menores a 20 minutos.

3.- A partir del estudio de investigaciones anteriores realizadas sobre el tema, se concluye que un análisis territorial como el presentado en esta investigación, puede ser un instrumento de planificación que apoye la toma de decisiones relacionadas con la localización adecuada de este tipo de equipamientos. Es decir, la propuesta metodológica complementa los avances que sobre la localización de este tipo de servicios actualmente se encuentran, pero observado desde el ofrecimiento de acceso por parte de la red de infraestructuras de transporte de una ciudad.

\section{AGRADECIMIENTOS}

Los investigadores quieren agradecer a la oficina de movilidad sostenible de la Universidad Nacional de Colombia sede Manizales y los estudiantes que hacen parte de está.

\section{REFERENCIAS}

Cardona, S., D. A. Escobar y C. A. Moncada, Evolución del número de pasajeros movilizados en los Sistemas estratégicos de transporte público en Colombia, Análisis Cuantitativo, Revista Espacios, 38(53) (2017)

Cardona, S., D. A. Escobar y C. A. Moncada, Comparison on Turn Costs in Accessibility Models. Case Study: Manizales, Colombia, http://doi.org/10.5539/mas.v12n8p59, Modern Applied Science, 12(8), 59 (2018)

Deo, N., Graph theory with applications to engineering and computer science, Prentice-Hall Inc. (1974)

Escobar, D.A., C.F. Urazán y C.A. Moncada, Análisis de Cobertura Urbana de los Nodos de Actividad Primaria Mediante un Estudio de Accesibilidad Territorial en Quibdó (Colombia), doi: 10.4067/S0718-07642017000500018, Información Tecnológica, 28(5), 177-190 (2017)

Escobar, D.A., J. Montoya y C.A. Moncada, Accessibility Analysis towards Urban Ecoparks as a Measure of Integral Environmental Planning - Case Study: Manizales, Colombia, http://doi.org/10.17485/ijst/2018/v11i21/122476, Indian Journal of Science and Technology, 11(21) (2018) 
Escobar, D.A., O. Tapasco y J. Giraldo, Medición de Desempeño del Sistema de Transporte Cable Aéreo de la Ciudad de Manizales en Colombia, usando Tres Enfoques: Analítico, Simulado y de Accesibilidad Urbana, http://doi.org/10.4067/S0718-07642015000600020, Información Tecnologica, 26(6), 199-210 (2015)

Gallego, D.F. y D. A. Suárez, Análisis Espacial De Las Estaciones De Bomberos En El Área Urbana Del Municipio Santiago De Cali Para El Año 2014, Estimación de Su Cobertura y Tiempo De Respuesta (2015)

Geurs, K.T., A. De Montis y A. Reggiani, Recent advances and applications in accessibility modelling. Computers, http://doi.org/10.1016/j.compenvurbsys.2014.09.003, Environment and Urban Systems, 49, 82-85 (2015)

Geurs, K. T. y J. Ritsema Van Eck. Accessibility measures: review and applications. Evaluation of accessibility impacts of land-use transportation scenarios, and related social and economic impact. RIVM Report, 787, 1-265 (2001)

Geurs, K. T. y B. van Wee, Accessibility evaluation of land-use and transport strategies: Review and research directions, http://doi.org/10.1016/j.jtrangeo.2003.10.005, Journal of Transport Geography, 12(2), 127-140 (2004)

Hansen, W.G., How Accessibility Shapes Land Use, http://doi.org/10.1080/01944365908978307, Journal of the American Institute of Planners, 25(2), 73-76 (1959)

Holguin, J.M., D.A. Escobar y C. A. Moncada, Access to Emergency Medical Services: An Urban Planning Methodology for the Generation of Equity, http://doi.org/10.5539/gjhs.v10n6p181, Global Journal of Health Science,10(6), 181 (2018)

Holguín, J.M., D.A. Escobar y J.A. Tamayo, Servicio de urgencias versus infraestructura de transporte. Un análisis de accesibilidad enfocado en las desigualdades sociales. Caso de estudio: Manizales, Colombia, http://doi.org/10.4067/S071807642017000200014, Información Tecnologica, 28(2), 125-134 (2017)

Ingram, D.R., The Concept of Accessibility: A search for an operational form, https://doi.org/10.1080/09595237100185131, Regional Studies, 5(2), 101-107 (1971)

Lazo, J. E., Metodología para la optimización de la cobertura de respuesta en la atención de bomberos en clave 0-11, Memoria para optar al Título de Ingeniero Civil Industrial, Facultad de Ingeniería, U. Andrés Bello, Santiago, Chile (2017)

Liu, N., B. Huang y M. Chandramoul, Optimal Siting of Fire Stations Using GIS and ANT Algorithm, doi:10.1061/(ASCE)0887-3801(2006)20:5(361), Journal of Computing in Civil Engineering, 20(5), 361-369 (2006)

Lucas, K., B. van Wee y K. A. Maat, A method to evaluate equitable accessibility: combining ethical theories and accessibility-based approaches, http://doi.org/10.1007/s11116-015-9585-2, Transportation, 43(3) (2016)

Matheron, G., Principles of Geostatistics, Economic Geology, 58, 1246-1266 (1963)

Moreno, S. L. y J.M. Jácome, Usos de información georreferenciada para prestación de servicios a la población: Una revisión de literatura, http://dx.doi.org/10.21138/GF.572, GeoFocus - Revista Internacional de Ciencia y Tecnología de la Información Geográfica, (20), 201-229 (2017)

Murtadha S.S., M.A. Ameer y otros tres autores, Assessment And Development of the Spatial Coverage of Fire Service in Nasiriyah City by Using Geographic Information Systems (GIS), International Journal of Civil Engineering and Technology, 9(7), 936-947 (2018)

Oliver, M.A. y R. Webster, Kriging: A method of interpolation for geographical information systems, http://dx.doi.org/10.1080/02693799008941549, Int. J. Geographical Infor. Systems, 4(3), 313-332 (1990)

Patrick, S.W., M.M. Davis, C.U. Lehmann y W.O. Cooper, Increasing incidence and geographic distribution of neonatal abstinence syndrome: United States 2009 to 2012, https://doi.org/10.1038/jp.2015.36, J. of Perinatology, 35(8), 650-655 (2015)

Perilla, D. J., D. A. Escobar y S. Cardona, New Transportation Infrastructure Impact in Terms of Global Average Access Intersection "La Carola" Manizales (Colombia) Case Study, https://doi.org/10.12988/ces.2018.812, Contemporary Engineering Sciences, 11(5), 215-227 (2018).

Pirie, G.H., Measuring Accessibility: A Review and Proposal, http://doi.org/10.1068/a110299, Environment and Planning A, 11(3), 299-312 (1979)

Savsar, M., Fire station location analysis in a metropolitan area, DOI: 10.1504/IJISE.2014.060134, International Journal of Industrial and Systems Engineering, 16(3), 365-381 (2014)

Simpson, T.W., T.M. Mauery, J. Korte y F. Mistree, Kriging models for global approximation in simulation-based multidisciplinary design optimization, http://doi.org/10.2514/3.15017, AIAA Journal, 39(12), 2233-2241 (2001)

Van Wee, B., Accessible accessibility research challenges, http://doi.org/10.1016/j.jtrangeo.2015.10.018, Journal of Transport Geography, 51, 9-16 (2016)

Younes, C., D. A. Escobar y J. M. Holguín, Equidad, accesibilidad y transporte. Aplicación explicativa mediante un análisis de accesibilidad al sector universitario de manizales (Colombia), http://doi.org/10.4067/S0718-07642016000300010, Informacion Tecnologica, 27(3), 107-118 (2016)

Zhang, W. y J.C. Jiang. Research on the location of fire station based on GIS and GA, doi:10.4028/www.scientific.net/amm.130-134.377, Applied Mechanics and Materials, 130, 377-380 (2012)

Zuluaga, J. y D. Escobar, Geomarketing Analysis for Shopping Malls in Manizales (Colombia), Accessibility approach methodology, Revista Espacios, 38(21), 20 (2017) 